\title{
Clickers y Exámenes Tipo Test, Herramientas Interactivas de Evaluación Contínua
}

Gumbau-Albert, M. ${ }^{\text {a }}$, García-Cárceles, B. ${ }^{\text {, }}$ Marín, A. , Pastor, JM. $^{\text {ac }}$ y Villagrasa, J. ${ }^{\text {d }}$ ${ }^{a}$ Universitat de València, Análisis Económico (mercedes.gumbau@uv.es, garcarbe@uv.es, jose.m.pastor@uv.es ), ${ }^{b} U$ niversitat de València, (Antonio.marin@uv.es ) y ${ }^{c}$ Universitat de València, (Jorge.villagrasa@uv.es ).

\begin{abstract}
In this article, clickers tool is presented as an educational device based on an Electrónic voting system that aims to foster interaction, debate and dynamism in the audience. Among its main advantages over other existing educational resources we can highlight its simplicity, variety of use and ease of implementation in PowerPoint ${ }^{\circledR}$ presentations.

Through this experience the use of the clicker system is tested to be used in multiple-choice exams. This tool facilitates time optimization, allows students to obtain immediate assessment of their results and contrast their individual level of knowledge against the class. Likewise, throughout this paper it is assessed the speed and reliability of the devices, options of integration in the continuous assessment process and the effect that this methodology has on students.

As a result, an incentive to the study is created among students who got worse results and an additional motivation for reaching the best notes are observed.
\end{abstract}

Keywords : clicker, teaching, innovation, interactivity, participation.

\section{Resumen}

En este artículo se presenta la herramienta clickers, un dispositivo pedagógico basado en un sistema de votación electrónica que tiene como objetivo fomentar la interacción, el debate y el dinamismo en la audiencia. Entre sus principales ventajas, con respecto a otros recursos educativos existentes, puede destacarse su sencillez, variedad de uso y facilidad de implementación en presentaciones de PowerPoint ${ }^{\circledR}$.

En este trabajo se presenta el uso del sistema clicker para realizar exámenes tipo test de evaluación contínua a las alumnas y los alumnos. Esta herramienta facilita la optimización del tiempo, permite que el alumnado obtenga evaluación inmediata de sus respuestas y posibilita el contraste de su conocimiento individual con el del resto de la clase. Asimismo, a lo largo de este trabajo se evalúa la rapidez y fiabilidad de los dispositivos, las distintas opciones de integración en el proceso de evaluación contínua de la 
asignatura y el efecto que tiene sobre los estudiantes la incorporación de dicha metodología.

Como resultado, se observa un incentivo al estudio de las personas que obtienen peor evaluación y una motivación adicional para las que alcanzan las mejores notas.

Palabras clave: clicker, docencia, innovación, interactividad, participación.

\section{Introducción}

La implantación del Plan de Bolonia en el curso 2010-2011, dentro del marco del Espacio Europeo de Educación Superior (EEES), supuso la puesta en práctica de los planes de convergencia de los sistemas universitarios europeos. El calado del proceso de homogeneización de competencias, títulos, etc. está siendo profundo y supone un cambio de enfoque a todos los niveles de la estructura universitaria y del ejercicio de la docencia. En la tabla 1 se recoge este cambio de paradigma en el enfoque de la docencia en educación superior:

Tabla 1. Cambio de paradigma en la docencia universitaria

\begin{tabular}{l|l|l|l}
\hline Dimensión & \multicolumn{1}{|c|}{ Antes } & \multicolumn{1}{c}{ En proceso } & \multicolumn{1}{c}{ ¿Futuro? } \\
\hline Docencia & $\begin{array}{l}\text { "Me enseñan" } \\
\text { Trabajo individual } \\
\text { Materiales }\end{array}$ & $\begin{array}{l}\text { Manual, pizarra y } \\
\text { "Aprendo" } \\
\text { Trabajo en grupo } \\
\text { Casos, problemas } \\
\text { relacionados }\end{array}$ & $\begin{array}{l}\text { "Nos ayudamos a aprender" } \\
\text { Coaprendizaje } \\
\text { Resolución de casos nuevos en } \\
\text { situaciones interdisciplinarias }\end{array}$ \\
Motivación & $\begin{array}{l}\text { Evaluación final } \\
\text { Examen) }\end{array}$ & Evaluación continua & $\begin{array}{l}\text { Autoevaluación y coevaluación } \\
\text { Evaluación 360 }\end{array}$ \\
Docente & $\begin{array}{l}\text { Aprender } \\
\text { clase magistral }\end{array}$ & $\begin{array}{l}\text { Adquirir autonomía para el } \\
\text { aprendizaje y confianza en la } \\
\text { resolución de problemas futuros } \\
\text { sin experiencia anterior }\end{array}$ \\
\hline
\end{tabular}

En este tiempo se ha abierto un intenso debate en torno a las posibilidades de la renovación metodológica con el fin de conseguir el cambio de paradigma buscado. Si bien la lección magistral sigue siendo el método predominante en el panorama universitario (Fernández March, 2006) se ha podido observar una creciente tendencia a la exploración de 
metodologías para el aprendizaje activo y colaborativo (García-Cárceles \& Román, 2012) (Villagrasa, Marín, Garcia-Carceles, \& Pastor, 2016) o del uso de las TIC y sus aplicaciones en la docencia o en el seguimiento y evaluación de los y las estudiantes (del Ramo \& López, 2008) (Pastor, 2009) (Villagrasa, Marín, \& Pastor, 2016). Esta tendencia se ha observado con indiferencia de las áreas de conocimiento y tipo de asignatura, encontrando ejemplos de aplicación en economía (García, García-Cárceles, \& Pavía, 2012), finanzas (Comeig, Jaramillo-Gutiérrez, \& Ramírez, 2011), medicina (Vilar, 2011), derecho (Garía, 2009) e ingeniería (García J. C., 2011), por citar algunos.

Así, se constata el hecho de que el uso de las tecnologías de la información y la comunicación (TIC) ofrece un sinfín de oportunidades para mejorar la docencia con independencia del perfil de la signatura. Si bien no se trata de implementar la tecnología "per sé" en el aula, pero sí se busca mejorar la experiencia del alumnado a través del uso de herramientas tecnológicas que situarían a las alumnas y los alumnos como protagonistas del proceso de aprendizaje (Salinas, 2004) (Llorenç, 2012).

En concreto, en el caso que nos ocupa, la cuestión a resolver puede considerarse una tipificación del problema de seguimiento y evaluación en asignaturas troncales. Se trata de materias con densidad en el contenido a transmitir, complejidad de los conceptos (en su mayoría totalmente nuevos para el alumnado) y cuya asimilación compromete el desarrollo de sus competencias en cursos sucesivos.

El tiempo en el que debe "comprimirse" el proceso de transmisión, asimilación, práctica y evaluación del conocimiento en estas asignaturas deja poco margen de maniobra para la dinamización a través de metodologías activas y de colaboración. A su vez, el aprovechamiento de las TIC en el seguimiento y evaluación de este tipo de asignaturas suponen una oportunidad a considerar.

En este sentido, en este trabajo se presenta la primera exploración realizada para el uso de los llamados dispositivos personales de respuesta para mejorar la realización de pruebas de evaluación tipo test en asignaturas con las características antes descritas con el objetivo de dinamizar su realización, facilitar su evaluación y motivar a los estudiantes en el seguimiento y profundización de la asignatura.

En los siguientes apartados se presenta la experiencia del uso de clickers y la aplicación TurningPoint ${ }^{\circledR}$ en una prueba tipo test de la asignatura Microeconomía del Grado de Economía en la Universitat de València. Se evalúa la rapidez y fiabilidad de los dispositivos, las distintas opciones de integración en el proceso de evaluación contínua de la asignatura y el efecto que tiene sobre los estudiantes la incorporación de dicha metodología.

Tras la realización de la experiencia puede concluirse que, bien implementada, la herramienta facilita la optimización del tiempo de preparación y ejecución de los exámenes, permite que el alumnado obtengan evaluación inmediata, además de situar el nivel de conocimiento individual a nivel global dentro de la clase. Como resultado, se observa un incentivo al estudio de aquellas personas que obtienen peor resultado y una motivación adicional para las que alcanzan las mejores notas.

(cc) EY-NC-ND 2016, Universitat Politècnica de València

Congreso In-Red (2016) 


\section{Objetivos}

Considerando las características propias de la asignatura, el objetivo principal es buscar alternativas que permitan mejorar el proceso de asimilación de conceptos para los alumnos y las alumnas (conseguir que sea más rápido y profundo), así como simplificar el seguimiento y la evaluación de la asignatura, reduciendo al mínimo el trabajo administrativo.

Por su parte, los objetivos concretos en el contexto de la metodología docente serían: evaluar la rapidez y fiabilidad de los dispositivos personales de respuesta, las distintas opciones de integración en el proceso de evaluación contínua de la asignatura así como el efecto que tiene sobre los estudiantes la incorporación de dicha metodología.

En relación a los estudiante, en primer lugar, se pretende que el alumnado mantenga el ritmo de estudio a lo largo del semestre. De esta forma, pueden asentar los conocimientos para avanzar en el de los siguientes temas de forma paulatina y reflexiva. Nuestro punto de vista es que sólo de este modo se logra que el alumnado llegue a entender la conexión entre la realidad económica y la estilización de los hechos en gráficos y modelos matemáticos que el profesor expone en clase.

En segundo lugar, se persigue que la realización del examen tipo test sea lo más dinámica posible, permitiendo la interacción entre estudiantes y docente en la sesión.

En tercer lugar, gracias a esta herramienta podemos disponer de resultados inmediatos y gráficos con las respuestas que el alumnado puede interpretar, ayudando a que cada uno pueda autoevaluar su desempeño individualmente y en el conjunto de la clase.

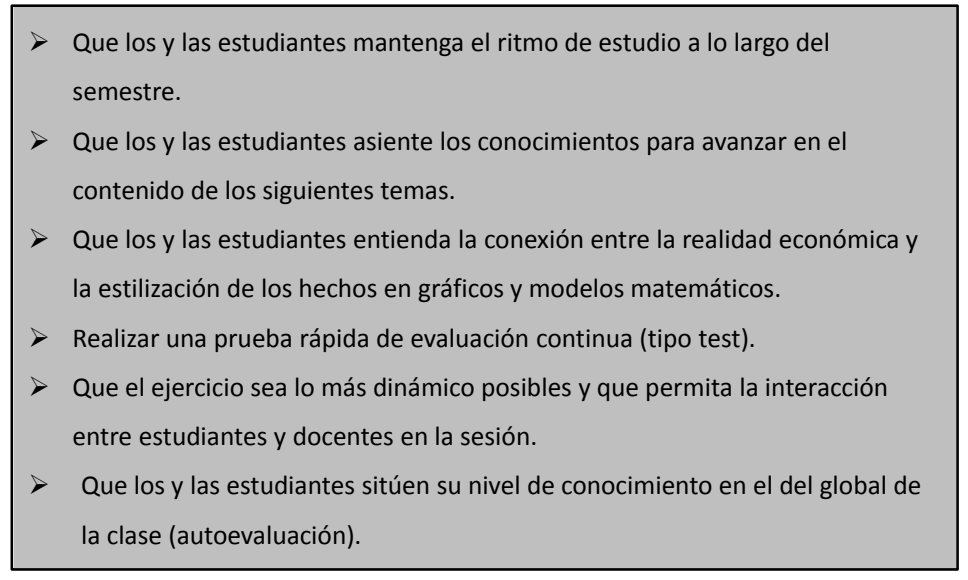

Fig. 1 Resumen de objetivos de la actividad

De la descripción de los objetivos antes citados se desprenden dos supuestos de partida:

1- La validez del formato de examen tipo test. El formato test se mantiene porque tiene notables ventajas, entre ellas destacan que su resolución requiere un ejercicio de síntesis por parte del alumnado, además de desarrollar su habilidad de profundizar en conceptos que de otra forma les pasan desapercibidos.

(cc) EY-NC-ND 2016, Universitat Politècnica de València 
2- Las posibilidades de mejora del formato anterior con los clickers. Los dispositivos personales de respuesta han sido ampliamente utilizados en el contexto de la docencia universitaria en sus distintos formatos (Pintor, Gargantilla, Herreros, \& López, 2014) (Villagrasa, Marín, Garcia-Carceles, \& Pastor, 2016), destacando su sencillez, variedad de uso y facilidad de implementación con materiales preexistentes.

La incorporación de este tipo de dispositivos en la Universitat de València se hizo efectiva en el curso 2014 y permitió su uso en actividades docentes desde entonces. En concreto, la solución puesta a disposición del profesorado gestiona el sistema de dispositivos personales de respuesta a través de clickers y de la aplicación TurningPoint ${ }^{\circledR}$. Entre las ventajas del aplicativo es de reseñar su uso intuitivo y la rapidez de obtención de informes pormenorizados del desempeño de cada estudiante en la elección de sus respuestas, además de su integración en el programa PowerPoint ${ }^{\circledR}$ que es el utilizado en la asignatura para la elaboración de las presentaciones.

\section{Desarrollo de la innovación: identificación del problema, plan de acción, implementación y resultado}

\subsection{Identificación del problema}

En la asignatura de Microeconomía, como en otras asignaturas troncales, sigue teniendo un papel relevante la clase magistral con método de evaluación clásico (prácticas entregables en la evaluación continua, realización de pruebas periódicas y examen final), dejando amplios espacios para la incorporación de métodos basados en las nuevas tecnologías que aumenten el protagonismo de los y las estudiantes en su proceso de aprendizaje, como en la mejora de la eficiencia de las tareas de evaluación.

\subsection{Plan de acción}

Se revisan los métodos de evaluación contínua de la asignatura y se recapacita en base a aquellas metodologías que se han mostrado válidas en el pasado y que son susceptibles de ser mejoradas con la incorporación de soluciones de base tecnológica.

Se identifica la solución de los dispositivos personales de respuesta disponible en la Universitat de València y se detecta la oportunidad de mejora en la realización de los exámenes tipo test.

\subsection{Implementación}

En cursos anteriores el uso de exámenes tipo test ha sido habitual en la evaluación continua, sin embargo se realizaban de una forma "clásica", es decir, entregando un ejercicio individual a cada estudiante que debía contestar dentro del tiempo estipulado durante una sesión de práctica o clase magistral. Debían esperar, entonces, a la corrección por parte del docente y los resultados se comunicaban de forma individualizada.

Se planifica una actividad "piloto" con uno de los grupos de la asignatura de Microeconomía para testar las hipótesis de mejora aplicando una solución combinada

(cc) EY-NC-ND 2016, Universitat Politècnica de València

Congreso In-Red (2016) 
utilizando los clickers y la herramienta TurningPoint $₫$. Esta actividad se implementa en los aularios de la Facultat d'Economia en la Universitat de València.

Se diseña el cuestionario con preguntas tipo test y se convoca una sesión práctica con el incentivo de mejorar la nota de la evaluación continua.

\section{Resultados: confrontación de los ejercicios tipo test de forma "clásica" frente al uso de los clickers}

Sin dejar de reconocer la validez que tienen los ejercicios tipo test en la evaluación, la incorporación de los clickers ha supuesto un cambio sustancial y positivo en la realización de los mismos. Entre las ventajas, podemos citar:

Primero: la herramienta TurningPoint ${ }^{\circledR}$ permite especificar el tiempo máximo que se puede dedicar a cada pregunta. Esto favorece el mantenimiento de un grado de concentración alto y constante en cada pregunta y a lo largo de todo el ejercicio.

$>$ Segundo: la herramienta TurningPoint ${ }^{\circledR}$ ofrece la respuesta correcta de forma inmediata al finalizar el tiempo previsto en cada pregunta, así como el resumen gráfico de las respuestas seleccionadas. De esta forma los alumnos y las alumnas pueden valorar la dificultad de la pregunta y su posición relativa en el conocimiento de la clase.

> Tercero: después de cada pregunta es posible dar las explicaciones que se consideren oportunas, teniendo en cuenta cómo ha sido el resultado de las "votaciones". Es en este momento es cuando el alumnado entiende detalles que de otra forma pasan desapercibidos y que son fundamentales en el razonamiento económico.

$>$ Cuarto: en un ejercicio de test clásico, el alumnado llega a conocer el resultado global de su examen, pero pierde conciencia de cuáles han sido las preguntas en las que ha cometido un error, a no ser que se haga una corrección pregunta por pregunta en las sesiones de prácticas. En este punto, el nuevo sistema es, simultáneamente, examen y corrección pregunta a pregunta, desarrollándose en el mismo espacio de tiempo que un examen test "clásico" sin contar la corrección.

> Quinto: se evita el tiempo de corrección (ya sea manual o mediante medios mecanizados) de los ejercicios de test "clásicos", lo que permite un uso más eficiente del tiempo por parte del docente.

\subsection{Valoración de la actividad por parte del profesorado}

A través de las distintas experiencias del uso de los clickers en el aula, se ha obtenido un procedimiento docente innovador y fundamental para el desarrollo de actividades de evaluación continua.

Se han observado las siguientes mejoras en la dinámica de los estudiantes tras su utilización:

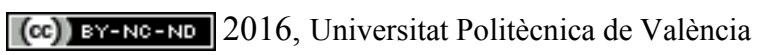


$>$ Por un lado, los estudiantes ajustan mejor las horas de estudio al cronograma previsto en la guía docente de la asignatura.

$>$ Además, el ejercicio actúa como autoevaluación para cada estudiante en tiempo real, es decir, en la misma sesión en la que se realiza el ejercicio, obtiene su evaluación en relación al conocimiento general del grupo.

$>$ Por otro lado, realizan un esfuerzo importante en la lectura de las preguntas, ya que éstas implican precisión en la respuesta $\mathrm{y}$, de esta forma, adquieren un conocimiento profundo del concepto que se les está preguntando. Entendemos que, de otra forma, estos conocimientos podrían pasarles desapercibidos.

> También se observa un incentivo al estudio de las personas que obtienen peor resultado y una motivación adicional para las que alcanzan las mejores notas.

$>$ Finalmente, este tipo de ejercicio asienta los conceptos necesarios que se necesitan para avanzar en el contenido de la asignatura.

\subsection{Valoración de la actividad por parte del alumnado}

Dado que se trata de una prueba piloto, se decidió no elaborar una encuesta formal. Si bien se recogió la opinión de los y las participantes en relación a los ejercicios utilizando la misma metodología de respuesta con clickers. Se hizo una pregunta de valoración global y una pregunta acerca de la puesta en común de resultados. En una escala del 1 al 5, todas las valoraciones se situaron entre 4 y 5 .

Entablando diálogo con el grupo de alumnos y alumnas al finalizar la sesión, se logró constatar que la resolución inmediata les permite comparar su conocimiento respecto al de sus compañeros y valorar su nivel en el tema concreto de forma que podían, a partir de ese momento, ajustar sus necesidades de estudio. La puesta en común, además, les permite entender claramente el concepto que se les exige dentro de la asignatura. Finalmente, se detectó que todas aquellas personas que habían pasado por alto en el estudio alguno de los conceptos planteados, después de la actividad se sentían más seguras a la hora de distinguir los aspectos más importantes del temario.

\section{Conclusiones}

En este trabajo se ha mostrado la experiencia del uso de los clickers en la realización de exámenes tipo test en la evaluación continua de las asignaturas de Microeconomía del Grado de Economía. Por sus características, esta asignatura troncal supone un reto para la implementación de metodologías activas centradas en el estudiante. Las herramientas docentes de base tecnológica suponen, por el mismo motivo, una fuente de oportunidades de mejora de la docencia en este tipo de asignaturas.

La experiencia pretende mejorar la metodología de evaluación tipo test tradicionalmente utilizada en la asignatura (desde que formaba parte en los planes de las licenciaturas ya en extinción). La constatada validez de este tipo de pruebas para la evaluación contínua, sin embargo, no está exenta de ser mejorada con nuevas herramientas. En concreto se evalúa el uso de clickers como sistemas individuales de respuesta para mejorar el dinamismo, la

(cc) EY-NC-ND 2016, Universitat Politècnica de València

Congreso In-Red (2016) 
interacción y la eficacia de los test como medida del progreso del alumnado en la evaluación continua.

La principal conclusión del estudio es simple, el uso de los clickers supone una mejora sustancial en la realización de un tipo de ejercicio esencial en el desarrollo de la evaluación continua: los exámenes tipo test. En particular, como elementos positivos se puede destacar el fomento de la interacción, el debate, el dinamismo en la audiencia, así como el mayor grado de atención y seguimiento de la sesión.

El feedback inmediato que reciben las participantes respecto a su nivel de conocimiento individual y en el contexto del grupo se revela como una fuente de motivación no anticipada en el diseño de la propuesta. Así, los y las estudiantes manifiestan mayor seguridad en la selección de contenidos relevantes que podrían estar pasando por alto.

Destaca también el bajo coste en la implementación de la herramienta así como en su aprendizaje, ya que se utiliza la aplicación PowerPoint $($ que es la habitualmente empleada para la preparación de los materiales en las explicaciones de la asignatura.

Es de reseñar que se produce una mejora en la eficiencia del uso del tiempo:

$>$ Por parte del alumnado, se logra la asimilación rápida y consistente de los conceptos tratados $\mathrm{y}$, por parte del profesorado, se "reinvierte" el tiempo de corrección del ejercicio en reforzar los conocimientos de la clase.

$>$ Asimismo, el sistema permite incorporar los resultados del ejercicio de forma casi automática al archivo de evaluación del profesor, reduciendo el tiempo dedicado a la gestión administrativa de los resultados.

\section{ANEXO: Detalle de la preparación y realización de la actividad}

El sistema clickers disponible en la Facultat d'Economia de la Unviersitat de València consiste en un programa informático llamado TurningPoint ${ }^{\circledR}$ que se instala en el ordenador del aula y que se ejecuta junto con la aplicación PowerPoint ${ }$, más unos mandos de respuesta individual y un receptor. Tanto la instalación del programa como su manejo resultan muy intuitivos, no requieren formación específica más allá de dedicar unas horas a comprender dónde se sitúa cada elemento: acceso al programa, manejo de listados de estudiantes, elaboración del archivo de preguntas, identificación de los mandos con cada estudiante y manejo de resultados del test.

\subsection{Acceso al programa:}

Una vez instalado el programa, se accede al mismo haciendo "click" en el icono del escritorio o seleccionando el programa en la lista de Windows ${ }^{\mathrm{TM}}$. Al hacerlo, se abre la

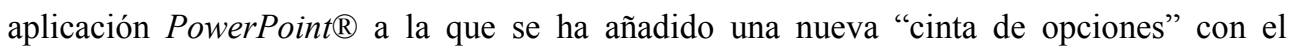
nombre "TurningPoint", en la que se muestran las herramientas de manejo del sistema clickers. 


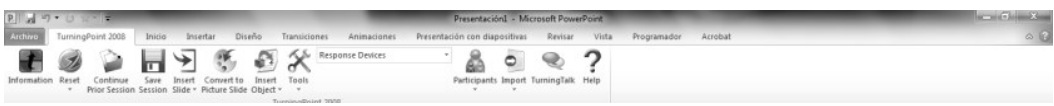

Fig. 2 Cinta de opciones generada por la herramienta TurningPoint ${ }^{\circledR}$ dentro de la aplicación

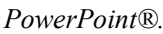

\subsection{Manejo de los listados de estudiantes:}

El sistema permite importar varios formatos de listados. En nuestro caso, los archivos han sido documentos de Excel ${ }^{\circledR}$. Dado que el programa TurningPoint $\AA$ incorpora un asistente para importar estos listados, la única dificultad consiste en respetar el orden en las columnas.

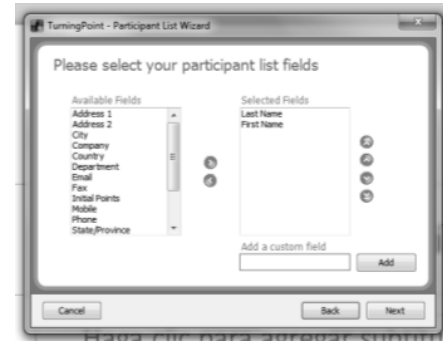

Fig. 3 Asistente para la importación de listados de alumnos.

\subsection{Elaboración del archivo de preguntas:}

El procedimiento es muy similar a la elaboración de una diapositiva de PowerPoint ${ }^{\circledR}$. Las diapositivas con preguntas deben generarse desde la "cinta de opciones de la herramienta TurningPoint ${ }^{\circledR}$ ", donde se dispone de varios formatos de pregunta. Una vez generada la plantilla de la diapositiva, puede ajustarse el formato libremente, aplicar fondos y diseños para que encajen dentro de las presentaciones habituales.

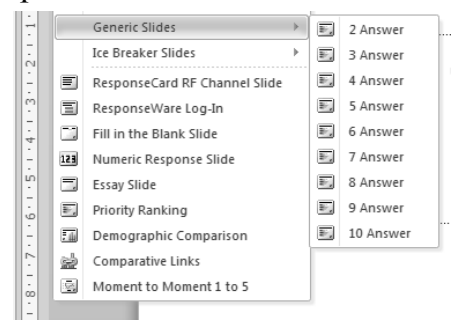

Fig. 4 Plantillas para la elaboración de presentaciones.

El ajuste de la respuesta correcta (sea una o varias), así como el número de preguntas y tipo de gráfico en los que se mostrará las contestaciones puede ajustarse de forma sencilla en la barra lateral derecha que se muestra en la ilustración.

(cc)) EY-NC-ND 2016, Universitat Politècnica de València 


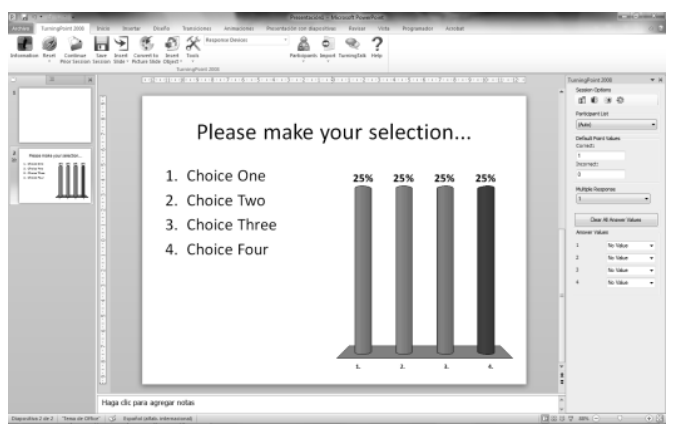

Fig. 5 Diapositiva tipo utilizando la herramienta TurningPoint $₫$ y los clickers.

\subsection{Identificación mando-estudiante:}

Una vez preparada la presentación, estamos listos para disfrutar de una sesión utilizando los clickers. La actividad se inicia repartiendo a cada estudiante un mando o dispositivo personal de respuesta para que pueda enviar las respuestas al sistema. Si bien es posible el uso de Smartphone en lugar de los mandos, nuestra experiencia se limita a éstos últimos. Su manejo es tan sencillo que no requiere explicación.

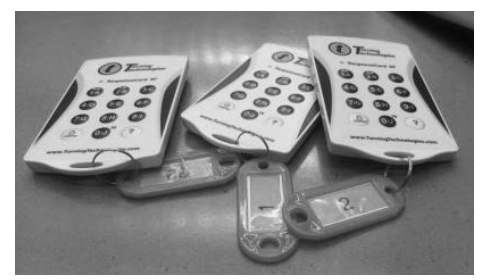

Fig. 6 Clickers.

Cada mando tiene un número de identificación único. Antes de iniciar la prueba tipo test, el programa TurningPoint ${ }^{\circledR}$ permite a cada estudiante identificarse de forma muy rápida con el mando que se le ha repartido para poder, de esta manera, identificar sus respuestas. Esto se realiza a través de un "click" sobre cualquier botón del mando.

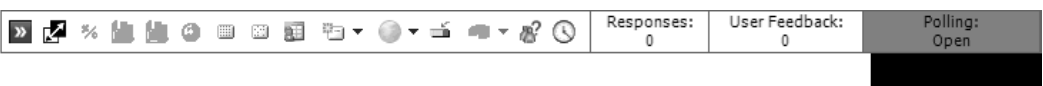

Fig. 7 Barra de control del sistema clickers durante la sesión.

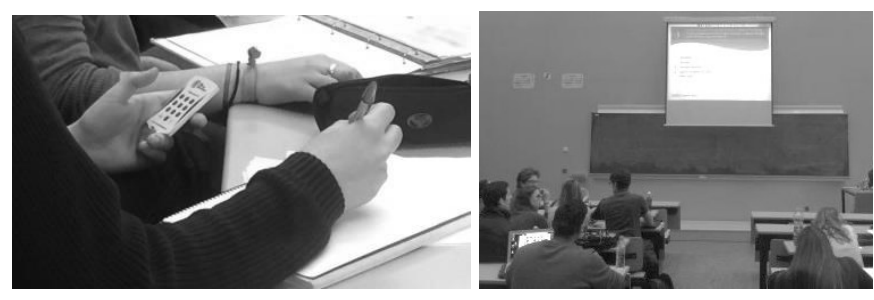

Fig. 8 Sesiones.

El archivo preparado con las preguntas (y las diapositivas explicativas, teóricas, etc.) se abre normalmente como un archivo PowerPoint ${ }^{\circledR}$ estándar. La diferencia es que cuando se 
llegue a una diapositiva de preguntas que permita la votación aparecerá una barra de opciones, indicando a los alumnos y las alumnas que ya pueden seleccionar sus respuestas (polling: Open). Al consumirse el tiempo establecido para contestar la pregunta, se muestra el gráfico con el número (o porcentaje) de personas que han seleccionado cada opción.

\subsection{Manejo de los resultados del test:}

Independientemente de que tras cada pregunta sea posible visualizar la frecuencia con la que cada respuesta ha sido seleccionada, el programa TurningPoint ${ }^{\circledR}$ guarda y genera informes con los resultados pormenorizados según estudiante, según pregunta, etc. La obtención de un listado con la nota global obtenida por cada participante es inmediata.

\section{Referencias}

Benito, A., \& Cruz, A. (2011). Nuevas claves para la docencia universitaria. Madrid: NARCEA, S.A. de Ediciones.

Comeig, I., Jaramillo-Gutiérrez, A., \& Ramírez, F. (2011). "Experimentos Interactivos para la Enseñanza de Economía y Finanzas". En Experiencias de Innovación Docente en Estadística (págs. 191-201).

del Ramo, J. J., \& López, N. (2008). Respondus, un programa para la creación y publicación de cuestionarios. @tic, revista d'innovació educativa(1),79-81.

Fernández March, A. (2006). Metodologías activas para la formación de competencias. Educatio siglo XXI(24), 35-56.

García, J. C. (2011). "Formación en Competencias y Adaptación de la Asignatura de Estadística Industrial al EEES en la Escuela Técnica Superior de Ingenieros Industriales de la Universidad Politécnica de Valencia". En Experiencias de innovación docente en estadística (págs. 39-48).

García, V., García-Cárceles, B., \& Pavía, J. M. (2012). "La teoría de juegos como herramienta para obtener datos empíricos sobre el comportamiento de los agentes económicos: una aplicación para la enseñanza en Estadística”. En Docencia en estadístcia. Experiencias de innovación (págs. 181-190). Valencia.

García-Cárceles, B., \& Román, M. V. (2012). "Tutorial dinàmics per a la docència en assignatures d'estadística: l'aprofitament de les TIC davant de la transformació del paradigma docent en el context de l'EEES". En Docencia en estadística. Experiencias de innovación (págs. 211-226). Valencia.

Garía, J. (. (2009). Miradas a la innovación: Experiencias de innovación en docencia del derecho. Valencia.

Llorenç, F. (. (2012). Tendencias TIC para el apoyo a la Docencia Universitaria. Madrid: Conferencia de Rectores de las Universidades Españolas (CRUE).

(cc) EY-NC-ND 2016, Universitat Politècnica de València

Congreso In-Red (2016) 
Pastor, J. M. (2009). Los formularios en línea como herramienta telemática para interactuar con los estudiantes. @tic revista d'innovació educativa, 3(79-83).

Pintor, E., Gargantilla, P., Herreros, B., \& López, M. (2014). KAHOOT en docencia: una alternativa práctica a los clickers., (págs. 322-329). Madrid.

Salinas, J. (2004). Innovación docente y uso de las TIC en la enseñanza universitaria. Revista Universidad y Sociedad del Conocimiento, 1(1), 1-16.

Vilar, E. (2011). Herramientas y métodos para la formación en radiodiagnóstico. Radiología, 3(53), 202-208.

Villagrasa, J., Marín, A., \& Pastor, J. (2016). La aplicación TurningPoint como herramienta de aprendizaje transformacional en los procesos educativos. @tic revista d'innovació educativa, Pendiente Publicacion.

Villagrasa, J., Marín, A., Garcia-Carceles, B., \& Pastor, J. (2016). The Clickers tool as a transformational learning process. INTED2016 Proceedings, 958-962.

Agradecimientos: Belén García Cárceles agradece la financiación del programa "Atracció de Talent" del Vicerrectorat d'Investigació i Política Científica de la Universitat de València. Los auotres agradecen la financiación por parte del Servei de Formació Permanent i Qualitat Educativa de la Universitat de València. 